\title{
Pasado, presente y futuro del proyecto Scielo en España
}

\author{
Por Cristina A. Fraga Medín, Cristina Bojo Canales y Silvia Hernández Villegas
}

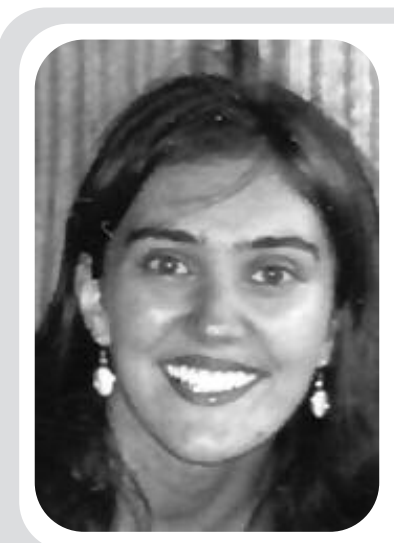

Cristina A. Fraga Medín es diplomada en biblioteconomía y documentación por la Universidad de A Coruña, licenciada en documentación por la Universidad de Salamanca y máster en información y documentación por la Universidad Carlos III de Madrid. Tras haber trabajado en otros centros, desde el año 2003 colabora en el desarrollo y aplicación del modelo SciELO-España, coordinado por la Biblioteca Nacional de Ciencias de la Salud.
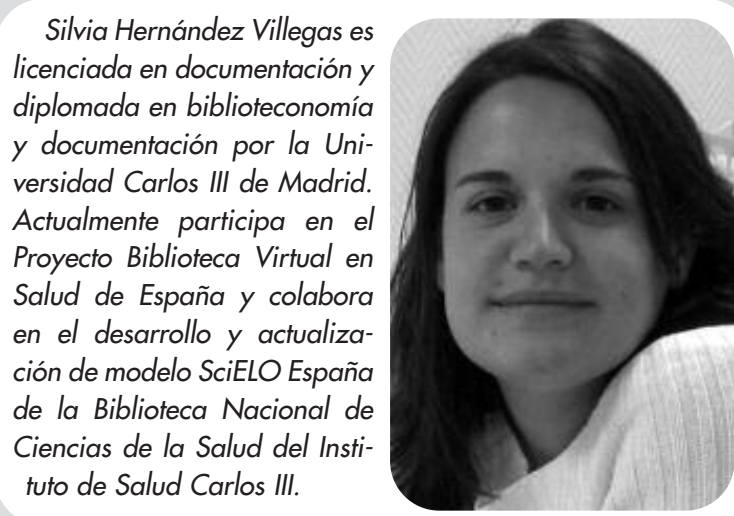

Resumen: El modelo SciELO (Scientific Electronic Library on-Line) es un repositorio open access centrado en la edición de contenidos científicos. Se describe su metodología, principales componentes y herramientas para búsqueda y recuperación de información. Asimismo, se analiza el caso concreto de SciELO-España, especializado en revistas españolas de ciencias de la salud: historia, evolución y perspectivas de futuro.

Palabras clave: SciELO, Open access, Metadatos, Literatura científica, Revistas científicas electrónicas.

Title: Past, present and future of the Scielo project

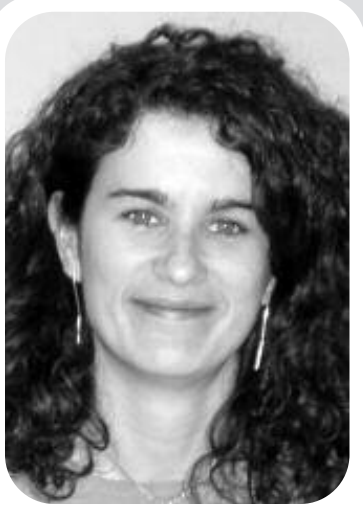
Cristina Bojo Canales es diplomada en enfermería y licenciada en documentación por la Universidad Complutense de Madrid. Trabaja en la Biblioteca Nacional de Ciencias de la Salud y desde hace dos años colabora en la coordinación y desarrollo del proyecto SciELO-España. in Spain

Abstract: SciELO (Scientific Electronic Library On-Line) is an open access repository focusing on scientific information publishing. This paper describes its methodology, main elements and tools for information search and retrieval. SciELO-Spain, which specialises in Spanish health science journals, is also described: its history, evolution and future perspectives.

Keywords: SciELO, Open access, Metadata, Scientific information, Scientific e-journals.

\section{Introducción}

EN LOS ÚLTIMOS AÑOS, LOS AVANCES en el desarrollo e implantación de sistemas de comunicación y difusión de la información basados en nuevas tecnologías han supuesto un paso definitivo y revolucionario en la comunicación científica. Este escenario ha permitido la puesta en marcha de un movimiento de universalización de la información en cuanto a conceptos tales como su acceso, difusión y transferencia.

En este contexto han aparecido distintas iniciativas que abogan por el acceso libre y gratuito a la información científica al mismo tiempo que por la normalización de la estructura de la información, de ma- nera que pueda ser interpretada por sistemas diversos de consulta y búsqueda.

Aunque el concepto es muy antiguo, la iniciativa de acceso universal y gratuito a la información -open access $(O A)$-, tal y como se enfoca en la actualidad, surgió en diciembre 2001 con la Budapest Open Access Initiative. Por otro lado, y muy vinculado a este movimiento, se ha desarrollado la norma que define la arquitectura para crear aplicaciones: OpenURL $L^{2,10}$.

La aplicación y desarrollo de estos dos proyectos permitirá gestionar de forma eficiente publicaciones periódicas a texto completo y poner en marcha modelos alternativos de publicación y comunicación científica basados en el acceso abierto ${ }^{8}$.

La Scientific Electronic Library Online (SciELO), que comenzó a fraguarse en 1997 en Brasil, aúna las dos técnicas. Es un modelo $O A$ en sí mismo, puesto que facilita artículos científicos de manera libre y gratuita a través de internet $y$, a la vez, produce documentos estructurados con metadatos que son susceptibles de enlace a través de pla-

10. Koutlas TC, Gaynor JW, Nicolson SC, Steven JM, Wemovsky G, Spray TL. Modified ultrafiltration reduces postoperative morbidity after cavopulmonary connection. Ann Thorac Surg 1997;64:37-43

[ Medline] [SciELO] [Lilacs]

Figura 1. Enlaces desde las referencias bibliográficas 
taformas OpenURL. El tratamiento de los archivos html con metadatos aumenta las posibilidades de búsqueda y recuperación, constituyendo una gran base de datos dinámica.

\section{Antecedentes del proyecto}

SciELO, parte integrante de la red de información y comunicación Biblioteca Virtual en Salud, es un proyecto resultado de la cooperación entre la Fundação de Amparo à Pesquisa do Estado do São Paulo (Fapesp) y el Centro Latinoamericano y del Caribe de información en Ciencias de la Salud (Bireme) de la Organización Panamericana de la Salud-Organización Mundial de la Salud (OPS/OMS).

Sus inicios (entre los años 1997-1998) consistieron en el desarrollo de una metodología apropiada para la edición, almacenamiento y organización en bases de datos de publicaciones científicas en formato electrónico. Entre marzo de 1997 y mayo de 1998 se puso en marcha un proyecto piloto con diez revistas científicas brasileñas que fue todo un éxito. Desde junio de 1998, y una vez constituido como un modelo de publicación de revistas científicas, el proyecto se encaminó hacia el desarrollo y puesta en marcha de los diferentes sitios y colecciones SciELO, siendo Brasil el país pionero.

\section{«El tratamiento de los archivos html con metadatos aumenta las posibilidades de búsqueda y recuperación, constituyéndose una auténtica base de datos dinámica»}

Hoy día están en pleno desarrollo 5 sitios SciELO geográficos (Brasil, Chile, Cuba, España y Venezuela) y 1 temático (Salud Públi$c a$ ). En fase de desarrollo existen ya

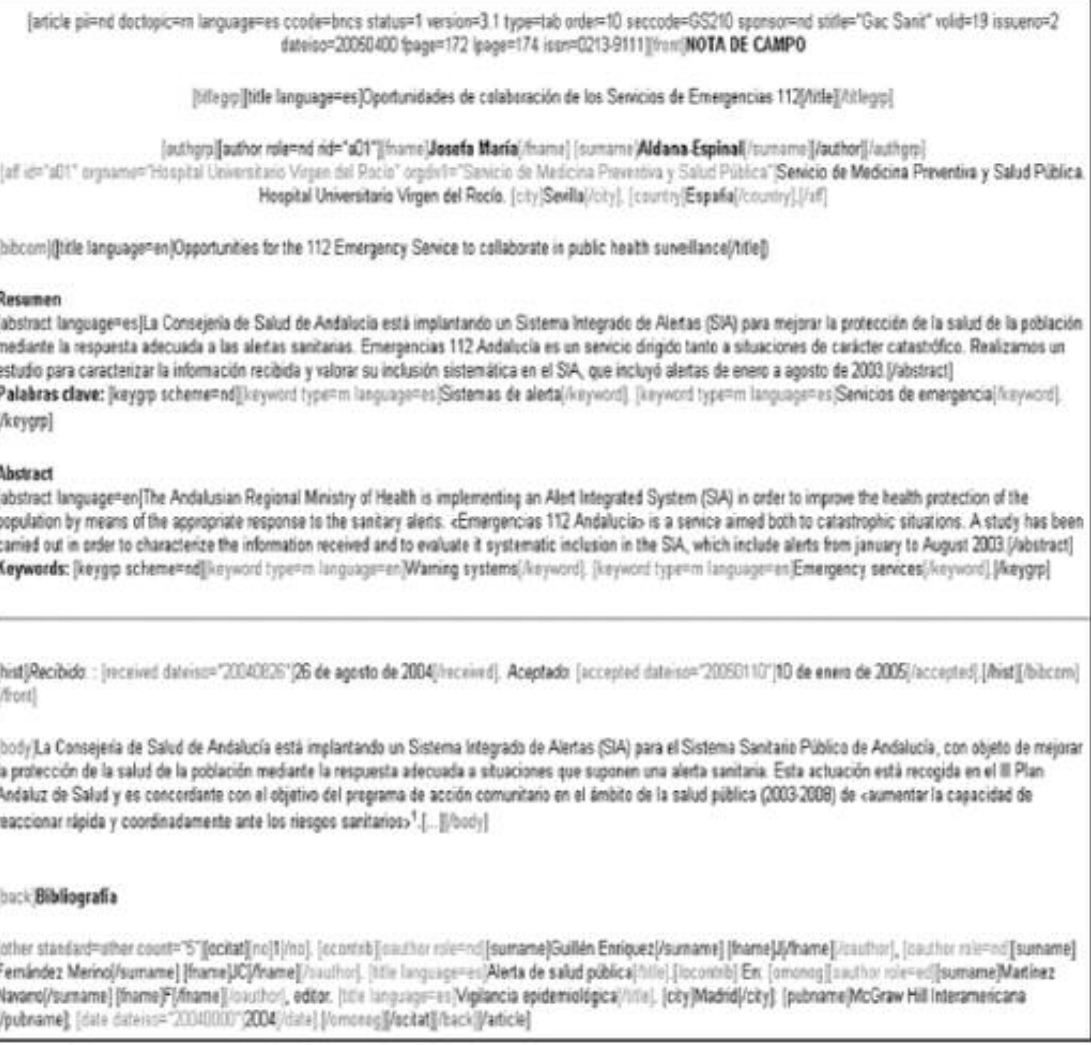

Figura 2. Artículo marcado según DTD-ScieLO Article 3.1.

7 proyectos de ámbito geográfico (Argentina, Colombia, Costa Rica, México, Portugal, Perú y Uruguay) y 2 especializados por tipos de documento (actas y tesis).

\section{El modelo de publicación electrónica Scielo}

Existen tres componentes fundamentales:

a. La metodología, que permite la publicación electrónica de revistas científicas, el establecimiento de bases de datos bibliográficas, el acceso a los textos completos y la recuperación de textos por su contenido. Incluye los criterios de calidad (que revisan aspectos de formato, contenido y difusión) establecidos para la evaluación, selección y permanencia de las revistas en los sitios SciELO ${ }^{14}$.

Entre las principales funciones de esta metodología encontramos:

- Medición de los accesos de las publicaciones editadas y obtención de estadísticas de uso.
- Medición del factor de impacto (FI) de las revistas dentro de la colección SciELO.

-Enlaces con otras fuentes de información (bases de datos y currícula de los autores).

Cabe señalar que estas utilidades no están presentes en todos los sitios SciELO existentes en la actualidad.

b. La aplicación de los citados mecanismos de acción, que da lugar a los diferentes sitios SciELO, permite la interoperatividad entre ellos. Todos están reunidos en una red integrada en el portal Red SciE$L O$, compuesta en la actualidad por los más desarrollados (Brasil, Chile, Cuba, España y el temático SciELO Salud Pública) y que da acceso a más de 200 revistas científicas de 20 materias distintas. http://www.SciELO.org

c. El desarrollo cooperativo mediante acuerdos de colaboración con los distintos componentes de la cadena de producción que permitan la difusión y evolución de este mo- 
delo: autores, editores e instituciones, bibliotecas y usuarios.

\section{«Hoy día están en pleno desarrollo 5 sitios SciELO geográficos (Brasil, Chile, Cuba, España y Venezuela) y 1 temático (Salud Pública)»}

La base de toda la metodología, y que la permite operar dentro de las iniciativas de $O A$ y ser objeto de enlace, son sus módulos de dtds, que se basan en las normas $I S O$ 8879-1986 (Sgml) e ISO 120831994 (Electronic manuscript preparation and markup). Este meta lenguaje describe la estructura y elementos que contienen las dtds definidas para el modelo $S c i E L O^{3,11}$.

Las dtds dividen el documento en varias partes. Cada una de ellas tiene asociadas una serie de etiquetas que permitirán "marcar" las secciones del documento (figura 2) que serán utilizadas por el sistema para generar la base de datos y permitirá la recuperación de la infor- mación y la interconexión con otras bases de datos.

El trabajo con las revistas se lleva a cabo mediante la colaboración entre las editoriales y el equipo SciELO. Las editoriales son las encargadas de enviar, cumpliendo los requisitos técnicos del sistema, los archivos en formato html de la revista y el equipo SciELO etiqueta cada artículo para poder introducirlo en el sistema y que éste lo procese para generar la base de datos.

\section{«El equipo SciELO etiqueta cada artículo enviado por las \\ editoriales para poder introducirlo en el sistema y que éste lo procese para generar la base de datos»}

Este desarrollo hace que actualmente SCiELO esté conectado a repositorios de $O A$ como el Directory of Open Access Journals ${ }^{4}$, bases de datos bibliográficas como el Índice Bibliográfico Español de Ciencias de la Salud (Ibecs), (Lilacs), Pubmed (por LinkOut), OCLC y WoS. http://www.doaj.org/

http://ibecs.isciii.es

http://bases.bireme.br/

http://www.ncbi.nlm.nih.gov/entrez/ query.fcgi

\section{Navegación por los sitios SciELO}

El hecho de que todos los sitios SciELO se generen con metodologías comunes hace que la información aparezca organizada del mismo modo, lo que facilita en gran medida su búsqueda y acceso.

Los sitios SciELO estructuran su contenido de manera individual en tres módulos:

a. Revistas, que da acceso al listado alfabético y de materias de las colecciones, aunque también se dispone de un formulario de búsqueda por título. Cada revista tiene su propio espacio que permite navegar por los números y hacer búsquedas exclusivamente en ella.

b. Artículos, desde el cual puede interrogarse la colección completa a través de los índices de autores y materias, y mediante los dos formularios de consulta, uno libre que permite hacer búsquedas senci-

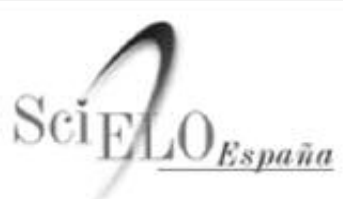

Seientific Electronic Library Online

ScIELO,org

criterios SCIELO

english

português

ayuda

acerca deste sitio revistas

lista alfabetica

lista por matena
búsqueda de títulos artículos

indice de autores

indice de materia

búsqueda de artículos

SciElo SciELO Espafía es una biblioteca virtual formada por una colección de revistas cientificas esparlolas de ciencias de la salud seleccionadas de acuerdo a unos criterios de calidad preestablecidos.

El proyecto SciELO es el resultado de la cooperación entre BIREME (Centro Latinoamericano y del Caribe de información en Ciencias de la Salud) y FAPESP (Fundación de Apoyo a la Investigación del Estado de Săo Paulo) y está siendo desarrollado en España por la Biblioteca Nacional de Ciencias de la Salud (BNCS), gracias al acuerdo de colaboración establecido entre la OPS/OMS y el Instituto de Salud Carlos III.

El principal objetivo es contribuir al desarrollo de la investigación, aumentando la difusión de la producción científica nacional, y mejorando y ampliando los medios de publicación y evaluación de sus resultados. 
llas por palabras y otro que posibilita la consulta por campos: palabras del título, autor, materia, resumen, año de publicación, tipo de artículo y afiliación del autor.

c. Informes, que permite elaborar estadísticas de uso del sitio y hacer cálculos bibliométricos (no disponible en todos los sitios) ${ }^{7}$.

Desde el portal Red SciELO pueden consultarse simultáneamente todos los sitios SciELO, tanto a través de un formulario libre como de la búsqueda de revistas por materias.

\section{SciELO-España}

Es un modelo $O A$ de edición electrónica basado en metadatos que da acceso a revistas científicas del área de ciencias de la salud editadas en España.

Nace en 1999 fruto del convenio de colaboración establecido entre la Organización Panamericana de la Salud y el Instituto de Salud Carlos III, instituciones que administran esta iniciativa a través de sus centros Bireme (Centro LatinoAmericano y del Caribe de Información en Ciencias de la Salud) y la Biblioteca Nacional de Ciencias de la Salud (BNCS).

Durante los años 1999-2000 se formó el equipo de trabajo y se implantó la infraestructura técnica para poder desarrollarlo. Era el momento de presentar esta iniciativa, hablar de los metadatos (tan desconocidos para muchos) y de convencer a los editores de las ventajas de publicar en abierto. Muchos veían en el $O A$ un peligro directo para sus intereses, pero los años han demostrado que aumenta la visibilidad y difusión de las publicaciones entre la comunidad científica. De hecho, la revista Nature publicó en octubre de 2003 un artículo sobre este proyecto en el que se explicaba cómo 5 publicaciones brasileñas indizadas por el ISI desde al menos 5 años y disponibles en SciELO desde al me- nos 2 habían aumentado su FI en un $132,7 \%$.

\section{«5 publicaciones} brasileñas indizadas por el ISI desde hace al menos 5 años y disponibles en SciELO desde hace al menos 2, han

$$
\begin{gathered}
\text { aumentado su FI en } \\
\text { un } 132,7 \% \text { » }
\end{gathered}
$$

$\mathrm{Al}$ mismo tiempo que se constituyó una colección inicial con cuatro títulos, comenzó la colaboración con la colección temática de SciELO-Salud Pública con la Revista española de salud pública, a la que seguiría posteriormente Gaceta sanitaria.

SciELO-España se presentó oficialmente como proyecto piloto en las IX Jornadas de información y documentación en ciencias de la salud, celebradas en Cáceres del 24 al 26 septiembre de 2001 y desde entonces es accesible a través de la Web.

\section{http://scielo.isciii.es/}

Durante los dos años siguientes (2001-2003), el trabajo del equipo se centró en la aplicación y perfeccionamiento de la metodología, además de continuar con la expansión de la colección. 2003 supuso la consolidación oficial ya que después de ser sometido (por parte de los técnicos de Bireme) a un exhaustivo análisis tanto de forma como de contenido, pasó a integrarse plenamente en la red Scielo. De nuevo, el anuncio oficial se produjo en unas jornadas profesionales, esta vez en Crics VI (Congreso regional de información en ciencias de la salud) celebrado del 6 al 9 de mayo de 2003 en Puebla (México) ${ }^{15}$.

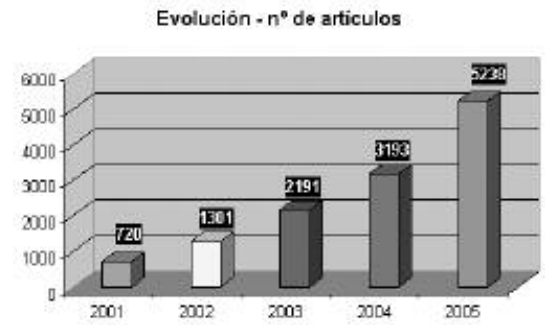

Figura 4. Evolución colección-número de artículos

En 2004 la colección experimentó el crecimiento más notable. Como puede verse en el gráfico de la figura 4 , el número de artículos en la colección ha ido incrementándose significativamente en los últimos años, siguiendo así en la primera mitad de 2005 tanto en número de revistas como en cantidad de artículos.

SciELO-España es un proyecto dinámico que espera incrementar su colección en los próximos meses con nuevos títulos de diferentes especialidades que se sumen a las 26 revistas de las 15 especialidades de ciencias de la salud ya existentes.

Tiene una filosofía eminentemente multidisciplinar y cooperativa, aunque en España se ha centrado en un principio en el área de ciencias de la salud, al ser el Instituto de Salud Carlos III la institución responsable y coordinadora del proyecto en nuestro país. Sin embargo, sería interesante poder establecer a escala nacional convenios con otras organizaciones interesadas en formar una red de revistas científicas electrónicas de otras temáticas.

\section{SciELO y PubMed}

El proyecto $S c i E L O$ no es ajeno a las demandas de los investigadores ni pretende ser una isla solitaria en medio del océano de la publicación científica. La literatura en español es necesaria pero no suficiente. La interconectividad con otros recursos resulta, además de beneficiosa, fundamental.

Desde hace un tiempo PubMed ha incorporado una opción que per- 
mite enlazar con el texto completo de los artículos que referencia: LinkOut, y los vínculos son mantenidos por los proveedores del texto completo. Actualmente, el texto completo de las revistas de SciELO-Brasil disponibles en PubMed está accesible a través de LinkOut. En SciELO-España ya se están haciendo gestiones para que esta posibilidad sea una realidad en un futuro cercano ${ }^{6}$.

Los beneficios son obvios: por un lado tenemos la referencia bibliográfica y por otro la posibilidad de acceder al texto completo.

\section{«Es necesario desarrollar otros recursos para evitar que revistas españolas con mayor factor de impacto no estén indizadas en el ISI y otras con menor FI sí lo estén»}

Además, la presencia de las revistas españolas en bases de datos internacionales es cada vez mayor. La calidad y visibilidad de nuestros títulos ha aumentado mucho en los últimos años, a pesar de que sigue siendo menor que la de las publicaciones del mundo anglosajón ${ }^{10}$. En el caso concreto de SciELO-España, de las 26 revistas disponibles 12 están en Pubmed, es decir, casi un 50\% de la colección'. Además, también están presentes en la base de datos Embase en donde, en 2003, se indizaban 13 de los 26 títulos.

\section{SciELO-España y el factor de impacto}

La visibilidad de una publicación y su impacto en la comunidad científica son temas que interesan mucho a quienes investigan. Entre

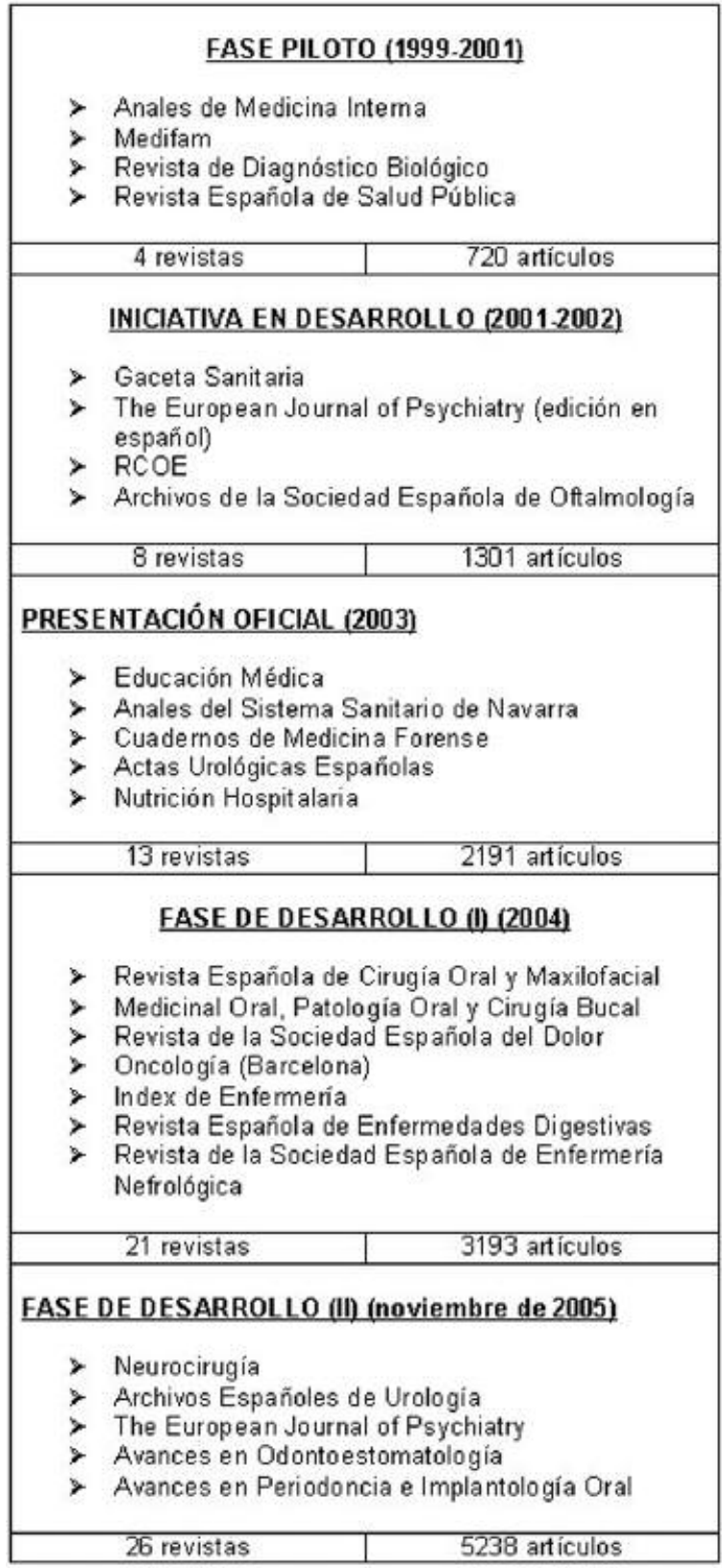

Figura 5. Evolución de la colección por años.

\begin{tabular}{|l|c|c|}
\hline & F.I. 2003 & F.I. 2004 \\
\hline NEUROCIRUGIA & 0.202 & 0.299 \\
\hline DIGESTIVAS & 0.348 & 0.593 \\
\hline EJP & 0.125 & 0.250 \\
\hline
\end{tabular}

Figura 6. Factor de Impacto 2003-2004

las herramientas disponibles para medir ese impacto destaca el JCR (Journal citation report) elaborado por el ISI. No vamos a entrar a discutir si esta herramienta es la más adecuada o no, o si los criterios para evaluar el impacto de una revista son suficientes o los más adecuados. Lo que sí diremos es que es necesario desarrollar otros recursos para evitar que revistas españolas con mayor factor de impacto no estén indizadas en el ISI y otras con menor FI sí lo estén.

Actualmente SciELO-España cuenta con tres revistas con FI medido por el ISI: Neurocirugía, Revista española de enfermedades digestivas y European journal of psychiatry (edición en español). El contrapunto positivo a esta reducida presencia es que si comparamos el FI de los años 2003 y 2004 observamos que casi se ha duplicado en los tres casos. Estos datos pueden ser un buen indicativo de la mejora de la consideración de las revistas de ciencias de la salud en el ámbito científico.

\section{Perspectivas de futuro}

- Ser un referente de calidad y fuente de información valiosa para los investigadores, aglutinando las revistas de ciencias de la salud más representativas de nuestro país.

-Establecer a escala nacional convenios con otras instituciones científicas con objeto de formar una red de revistas científicas electrónicas.

- Contribuir al desarrollo de una herramienta válida para la medición del FI de las revistas españolas.

- Mejorar la metodología, adaptándola a la realidad española y evolucionar haciendo uso de las ventajas que ofrece el entorno web implementando sistemas multimedia.

- Consolidar SciELO-España como una herramienta vinculada al movimiento $O A$ y Open $U R L$.

\section{Bibliografía}

1. Alonso, Wladimir J.; Fernández-Juricic,

Esteban. "Regional network raises profile of lo- 
cal journals". En: Nature, 2003, January, v. 31, n. 415, pp. 471-472.

2. Berlin declaration on open access to knowledge in the sciences and humanitites. Berlín, 20-22 de octubre de 2003. Consultado en: 0205-05.

http://www.zim.mpg.de/openaccess-berlin/ berlindeclaration.html

3. Bireme-OPS/OMS, Fapesp. SciELO-Scientific Electronic Library Online. Documentación del módulo dtd versión 3.1. São Paulo: BiremeOPS/OMS, Fapesp, octubre de 2000. Consultado en: 19-07-05.

http://www.scielo.org/dtd/dtd_scielo_ es.pdf

4. Directory of open acess journals (Doaj). Consultado en: 20-07-05.

http://www.doaj.org

5. ISI Web of Knowledge (WoK). Consultado en: 04-07-05.

http://isi02.isiknowledge.com/portal.cgi/

6. National Institutes of Health. "LinkOut home page". Consultado en: 11-07-05.

http://www.ncbi.nlm.nih.gov/entrez/linkout/

7. Martín Pastor, Belén. "Presentación y demostración práctica del modelo de publicación electrónica SciELO". En: VII Jornadas de informática sanitaria de Andalucía, 2001.
8. Melero, Remedios. "Acceso abierto a las publicaciones científicas: definición, recursos, copyright e impacto". En: El profesional de la información, 2005, v. 14, n. 4, pp. 255-266.

9. National Institutes of Health. "List of journals indexed for Medline 2005". Consultado en: 11-07-05.

ftp://nlmpubs.nlm.nih.gov/online/journals/ ljiweb.pdf

10. Open Society Institute. "Budapest Open Access Initiative". Consultado en: 29-04-05.

http://www.soros.org/openaccess

11. Packer Laerte, Abel; Biojone Rocha, Mariana ; Irati, Antonio M., [et al]. "SciELO: una metodología para la publicación electrónica".

En: Revista española de salud pública, 2001, v. 75, n. 4, pp. 291-312.

12. Alastruey, Mercedes; Calvo Tello, María; Pablos Martín, María de; Martín Pastor, Belén. "Biblioteca Nacional de Ciencias de la Salud: proyectos en marcha y proyección de futuro". En: IX Jornadas de información y documentación en ciencias de la salud, 2001.

13. Red SciELO. Consultado en: 08-06-05 http://www.scielo.org

14. SciELO, un modelo para publicación electrónica en países en desarrollo. Documento de trabajo. Consultado en: 20-07-05. http://www.scielo.org/model_es.htm

15. Veiga de Cabo, Jorge. "SciELO España". Sesión Especial. En: Congreso regional de información en ciencias de la salud, Crics-VI. Reunión de coordinación regional de la BVS, 2003. Consultado en: 08-06-05.

http://crics6.bvsalud.org/crics6/program/docs/ es/Scielo_es.ppt

16. Veiga de Cabo, Jorge; Martín Pastor, Belén; Calvo Tello, María; Pablos Martín, María de. "El modelo SciELO y su contribución a la difusión de las revistas de ciencias de la salud españolas". En: RCOE, 2003, v. 8, n. 1, pp. 6772.

17. Veiga de Cabo, Jorge. "La Biblioteca virtual en salud (BVS): una apuesta por la difusión de la producción científica española y latinoamericana en colaboración con la OPS/OMS", En: Revista española de salud pública, 2001, v. 75, n. 4, pp. 277-280.

Cristina A. Fraga Medín, Cristina Bojo Canales, Silvia Hernández Villegas, Biblioteca Nacional de Ciencias de la Salud. cbojo@isciii.es cristinafraga@isciii.es silviahernandez@isciii.es 